\title{
The Design and Development of Mathematics MicroLecture for Elementary School
}

\author{
Cuibai Li \\ College of Elementary Education \\ Hainan Normal University \\ Haikou, Hainan, China \\ 405900956@qq.com
}

\author{
Lifang Liu \\ College of Elementary Education \\ Hainan Normal University \\ Haikou, Hainan, China
}

\begin{abstract}
With the advancement of technologies, MicroLecture and Micro Video become more and more popular in elementary school because it's short and easy to learn. The article introduces the definition, type and related concept of MicroLecture. Then, the paper describes the strategies of instructional design and development of mathematics MicroLecture for primary school, combining with the example of Micro-Lecture of mathematics.
\end{abstract}

Keywords-Micro-Lecture; flipped classroom; mathematics; design strategy

\section{INTRODUCTION}

Since 2013, Micro-Lecture competitions grow up in the domestic. In China's Micro-Lecture Network, there have received more than 1900 participating video works of teachers and students, ranging from elementary school to high school in all the period, including from Number of language, math, science, politics, history, politics, history, music, art and etc.

\section{RELATED CONCEPTS OF MiCRO-LECTURE}

\section{A. The concept of Micro-Lecture}

With the development of Micro-Lecture, concept of Micro-Lecture becomes the focus of discussion. One of the most representative is Hu Tiesheng teacher who said " MicroLecture is instructional video as the main carrier, in accordance with requirements of the new curriculum standard and teaching practice, reflecting the teachers for a knowledge point in the process of classroom teaching or teaching links and teaching and learning activities of combination of various teaching resources."

\section{B. The Type of Micro-Lecture}

Micro-Lecture as a new type of teaching resources, the classification of Micro-Lecture can be attributed to the following aspects.

- According to the classification of teaching methods: Micro-Lecture may be divided into micro teaching class courseware, class discussion courseware, inspired Micro-Lecture, presentations, Micro-Lecture, practice courseware and etc.
- According to the categories of teaching method, Micro-Lecture may be divided into class micro lesson before class, new lesson to import Micro-Lecture, understanding knowledge of the Micro-Lecture, strengthen practice Micro-Lecture, knowledge development Micro-Lecture.

- According to the categories of use technology: MicroLecture may be divided into PPT of Micro-Lecture, record the screen video , electronic whiteboard, micro video lesson of Micro-Lecture, drama class.

\section{ThE Design StRATEgIES OF MATHEMATICS MICROLECTURE FOR ELEMENTARY SCHOOL}

\section{A. Basic Principles of Instructional Topic}

- The subject should be closely around the new curriculum syllabus, built around specific knowledge and teaching links, goals must be clear, the content must be pay attention to actual effect, selected topic to have typicality.

- The instructional content. When describing the chosen formula elements such as text, symbols, is to meet the national standards, The instructional content can't violate copyright, others cannot have sensitive content, at the same time when writing an article is science, the structure is complete, the logic is clear.

- $\quad$ The video is specification which is making video well and infectious voice. And making video should be to choose the appropriate method, time should not be more than 10 minutes, video recording is to clear picture, sound and animation which should reflect the teaching goal.

- Teaching activities should be to achieve the teaching goal. It can solve problems in the teaching or school, should will be able to improve the students' ability of innovation, understanding the main target. At the same time, the teaching process should be diversified, easily understood to improve students' learning enthusiasm and initiative. 
B. The design of Micro-Lecture of elementary school mathematics for "Area of Parallelogram"

- Analysis of instructional material. The area of a parallelogram is one version of chapter 6 of the first quarter for fifth grade, students learn the concept of parallelogram, properties and calculation formula on the basis of the area of square and rectangular.

- Micro goal. Through the lesson, students understand and use the formula to calculate the area of the parallelogram, formula the area of a parallelogram, solving practical problems.

- Teaching difficult point. The important points of teaching is understanding the area of the parallelogram calculation formula and using the right formula to calculate the area of parallelogram.

- Teaching process.(1) First of all, according to the practical problems in life, show the scene graph, ask questions (Who is kind of the plot of ground more? How to compare the size of the two plots of land?).(2) Activities of teaching new lesson. Count the squares, compare the size of the two plots of land and fill in the form of (two pieces of land area is the same).Leading to the area of the parallel quadrilateral and rectangular what kind of relationship is the key of the problem.(3) Guiding process of inquiry. Students have found two area of the same imagination, student exchanges and make use of the existing study actual hands-on, is obtained by using the conversion of parallelogram and the relationship between the rectangle area calculation formula, summed up the formula for the area of the parallelogram.(4) Practice and Exercises. Let students use the area of the parallelogram calculation formula to solve examples and the practical problems in life, to improve understanding of parallelogram area calculation formula.(5)Summary. Students conlude the calculating method of the area of the parallelogram .

\section{The Production Strategy of Micro-Lecture of ELEMENTARY SCHOOL MATHEMATICS}

\section{A. Basic Method}

Methods ofMicro-Lecture production usually have four kinds of method.

- Methods of camera + blackboard or electronic whiteboard: first designing teaching plan according to the micro subject, forming the lesson plan. Then using the blackboard in the teaching process, the whole process will be recorded with a video camera.

- Mobile phone + white paper. Using a mobile phone on pen paper combined with the operation, the writing process. The process includes: first, according to the micro subject design teaching plan, forming the lesson plan; Then pen calculus on the white paper, drawing, mark way according to the teaching process, such as mobile phone synchronization shooting. Finally it is editing of the video.
- $\quad$ PPT+ screen recording software PowerPoint Methods: making PowerPoint screen recording.The process includes that first making a PPT courseware according to the micro lesson topic. And then install software Camtasia Studio on the computer and open the PPT to choose the appropriate screen size, click the button of record, start recording. Finally it is clips and editing video later.

- The professional class production software + handwritten device Methods: through the "platform" Micro-Lecture production system for teaching demonstration, and simultaneously. Process: first, according to the micro subject design teaching plan, form the lesson plan; Then using micro platform software to do all kinds of layout of teaching object, and through the production system synchronization recorded: finally is late video processing.

\section{B. Making Skills}

The production skills of PPT includes several aspect.

- Avoiding the teacher according to the content of the PPT on scripted read it out. Core content should be instructive and sense of suspense, make students interested in the content of the PPT.

- The design of PPT layout is reasonable. PPT background should choose reasonable color.PPT content words to right size, color should be brighter, to facilitate students to watch.

- $\quad$ The PPT production must have the reasonable effects of animation. PPT is more beautiful, it attracts more students' attention. Appropriate animation effects can display content well, help students to understand.

- The recording techniques is reasonable.(1) Recording first should choose the appropriate instrument.(2) The record place should be a quiet place, preventing from noise. But it can not choose the empty place, prevent have echo(3) The voice of the lecture is nice. If the sound is too small or too large, adjust it.(4) The rhythm of language can not too fast or too slow.

In conclusion, as a new type of teaching resources, MicroLecture is an important supplement of traditional classroom teaching. Working for Micro-Lecture, is not only mastering methods and techniques of Micro-Lecture production, but it is most important that the teachers have understood students in the heart, although Micro-Lecture is for without classroom, but in the Micro-Lecture, teachers must have a student in the heart. So teaching skills of teachers is still most important in Micro-Lecture.

\section{Acknowledgment}

The article is supported by 2010 Humanities and Social Science Foundation of Ministry of Education of China (Grant No. 10YJC880063) and 2015 Instructional Reform Project of Higher Education Foundation of Hainan Educational Committee (Grant No. Hnjg2015ZD -7). 


\section{References}

[1] YouXiaoFang Tan Hui, Wang Ling, Yang Yujuan, fang ouyang, Wang Zhenwei, li added,"Primary and secondary schools in Shanghai multimedia use,”Journal of School Health in China,2013.11.

[2] Sammi,"Micro - network era of a new kind of constructivism teaching method,”Journal of Nanjing Industry Professional Technology Institute, 2013.3.

[3] Huang Yanqing, "Turn the classroom teaching design pattern study, ”.Journal of Software ,2013 .6.

[4] Hu Tiesheng,"Primary and secondary school construction and application of Micro-Lecture difficult problem dialysis,” Elementary and Middle Schools Information Technology Education, 2013.4 .

[5]Jia-hou li, "The meaning and development of the Micro-Lecture," Journal of Information Technology Education of Primary and Secondary Schools,2013 .4.
[6] Jian-li Jiao, “Micro Lesson and Its Application and Influence, ”Journal of Information Technology Education of Primary and Secondary Schools, 2013.4.

[7] Liang Leming Cao Qiaoqiao, Bao-hui Zhang, "Micro Course Design Pattern Research, Based on the Contrastive Analysis of Micro Course at Home and Abroad,"Research on Open Education, 2013.1.

[8] Kezhong Guan, “Micro course,’Information Technology Education in China, 2011.17

[9] Tian Qiuhua, "Miniature Curriculum and Its Development Strategy ,"Journal of Curriculum, Teaching Material and Teaching, 2009.5.

[10] Ceng Wenjie, "Miniaturization: deepening the road on the development of school-based curriculum, "Journal of Education Development Research,2009.4. 